\title{
Experimental overview of Generalized Parton Distribution results from HERMES
}

B. Zihlmann, and HERMES Collaboration

Citation: AIP Conference Proceedings 1149, 597 (2009); doi: 10.1063/1.3215718

View online: https://doi.org/10.1063/1.3215718

View Table of Contents: http://aip.scitation.org/toc/apc/1149/1

Published by the American Institute of Physics

Articles you may be interested in

Hadron Formation in Semi-Inclusive DIS on Nuclei at HERMES

AIP Conference Proceedings 1149, 690 (2009); 10.1063/1.3215738

Future GPD Measurements

AIP Conference Proceedings 1149, 579 (2009); 10.1063/1.3215714

\section{Conference Proceedings}

\section{Get $30 \%$ off all print proceedings!}

Enter Promotion Code PDF30 at check 


\title{
Experimental overview of Generalized Parton Distribution results from HERMES
}

\author{
B. Zihlmann \\ Jefferson Lab, 12000 Jefferson Avenue, Newport News, VA 23606 \\ ON BEHALF OF THE HERMES COLLABORATION
}

\begin{abstract}
Over the course of more than a decade the HERMES experiment has accumulated a wealth of data with electron and positron beams on various gaseous targets from Hydrogen up to Xenon. In addition, the beams and targets can be polarized. This data set is viewed in the context of Generalized Parton Distributions, a theoretical formalism with an explicit three dimensional view of the structure of the nucleon. It provides a link between experimental observables and the total angular momentum of the quarks in the nucleon.
\end{abstract}

Keywords: GPD, DVCS, Nucleon structure

PACS: $21.10 . \mathrm{Hw}, 25.30 . \mathrm{Rw}, 25.30 . \mathrm{Hm}$

\section{INTRODUCTION}

Hard exclusive reactions in lepton scattering can be expressed in terms of Generalized Parton Distributions (GPDs) $[1,2,3]$. GPDs are the probability amplitude for emitting a quark with longitudinal momentum fraction $x+\xi$ and absorbing a quark with longitudinal momentum fraction $x-\xi$ (fig. 1). The parton distribution functions (PDFs), known from inclusive and semi-inclusive deep-inelastic scattering, and the nucleon elastic form factors (FFs) are special limiting cases and moments of GPDs, respectively [2]. FFs are derived from elastic scattering experiments and describe the transverse distribution of the partons inside the nucleon, while PDFs describe the longitudinal momentum distribution of these partons. The combination of these two aspects of the nucleon structure is contained in the GPDs thereby providing a three dimensional picture of the nucleon $[4,5,6]$. In addition, a relation between the second moment of the GPDs $H$ and $E$ and the total angular momentum of the quarks in the nucleon is provided by the Ji relation [2]. The GPD $H^{q}(x, \xi, t)$ represents the unpolarized nucleon helicity non-flip GPD, while $E^{q}(x, \xi, t)$ represents the unpolarized nucleon helicity flip GPD. The Ji relation sparked a growing interest in measuring hard exclusive processes in lepton scattering. In this context the HERMES experiment [7] is well suited to measure azimuthal asymmetries of such reactions with its large acceptance using polarized electron or positron beams in combination with a longitudinal or transversely polarized Hydrogen target or with unpolarized nuclear targets like Deuterium, Nitrogen, Neon, Krypton and Xenon. 


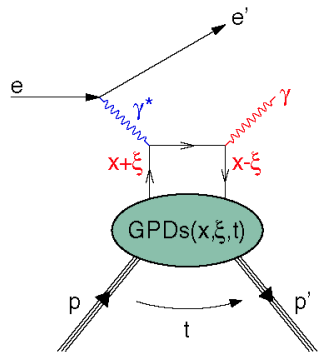

FIGURE 1. Handbag diagram of the DVCS process.

\section{DEEPLY VIRTUAL COMPTON SCATTERING}

Deeply Virtual Compton Scattering (DVCS) is the theoretically least complicated exclusive reaction to access GPDs. DVCS is the process where the virtual photon is absorbed by the struck quark which subsequently radiates a real hard photon before recombining with the spectator quarks to form the final state nucleon, as is depicted in the handbag diagram of figure 1. Because this reaction has the same final state as the Bethe-Heitler $(\mathrm{BH})$ process where the photon is radiated by the lepton beam particle rather then the struck parton (see figure 1), the cross section

$$
d \sigma \propto\left|\mathscr{T}_{B H}\right|^{2}+\left|\mathscr{T}_{D V C S}\right|^{2}+\left(\mathscr{T}_{B H}^{*} \mathscr{T}_{D V C S}+\mathscr{T}_{D V C S}^{*} \mathscr{T}_{B H}\right)
$$

involves an interference term $\mathscr{I}=\mathscr{T}_{B H}^{*} \mathscr{T}_{D V C S}+\mathscr{T}_{D V C S}^{*} \mathscr{T}_{B H}$ that allows direct access to the DVCS amplitude and the phase. The individual contributions to the cross section can be expressed in terms of series of Fourier components and is explicitly given in equation 2 up to twist-3 as

$$
\begin{aligned}
\left|\mathscr{T}_{B H}\right|^{2} & \propto c_{0}^{B H}+\sum_{n=1}^{2} c_{n}^{B H} \cos (n \phi)+s_{1}^{B H} \sin (\phi) \\
\left|\mathscr{T}_{D V C S}\right|^{2} & \propto c_{0}^{D V C S}+\sum_{n=1}^{2} c_{n}^{D V C S} \cos (n \phi)+\lambda s_{1}^{D V C S} \sin (\phi) \\
\mathscr{I} & \propto \pm\left(c_{0}^{I}+\sum_{n=1}^{3} c_{n}^{I} \cos (n \phi)+\lambda \sum_{n=1}^{2} s_{n}^{I} \sin (n \phi)\right)
\end{aligned}
$$

for an unpolarized nucleon target where $\phi$ is the azimuthal angle between the lepton scattering plane and the photon production plane as defined by the virtual and real photon in DVCS. The term $\lambda$ indicates the beam polarization and the term \pm indicates the beam charge for electron or positron beams. 


\section{Azimuthal Asymmetries}

To gain knowledge about the GPD $H$, one can use data taken with and unpolarized target and form azimuthal asymmetries with respect to either the beam charge or the beam polarization. This projects out different Fourier components of the interference term that depend on the real or imaginary part of the Compton Form Factor $\mathscr{H}$ [8][9]. The CFF $\mathscr{H}$ is a convolution of the GPD $H$ and the hard scattering kernel. Explicitly, the beam charge asymmetry (BCA) exhibits a $\cos (\phi)$ modulation of the azimuthal asymmetry proportional to the real part of $\mathscr{H}$, while the beam spin asymmetry (BSA) exhibits a $\sin (\phi)$ modulation of the azimuthal asymmetry and is proportional to the imaginary part of the $\mathscr{H}$. In a combined analysis of data with polarized electron and positron beams the ratio of the beam-polarized cross section to the unpolarized cross section can be written as a sum of asymmetries

$$
\frac{\sigma_{L U}}{\sigma_{U U}}=1+e_{l} \cdot A_{C}(\phi)+P_{b} \cdot A_{L U}^{D V C S}(\phi)+e_{l} \cdot P_{b} \cdot A_{L U}^{I}(\phi) .
$$

The first letter in the subscript of $\sigma_{X Y}$ refers to the beam polarization state while the second letter refers to the target polarization state using $U$ for unpolarized and $L$ for longitudinally polarized. The sign of the lepton beam charge is represented by $e_{l}$ and the beam polarization by $P_{b}$. The asymmetries are calculated from the data as

$$
\begin{aligned}
A_{C}(\phi) & =\frac{Y^{+\rightarrow}-Y^{-\rightarrow}+Y^{+\leftarrow}-Y^{-\leftarrow}}{Y^{+\rightarrow}+Y^{-\rightarrow}+Y^{+\leftarrow}+Y^{-\leftarrow}} \\
A_{L U}^{D V C S}(\phi) & =\frac{Y^{+\rightarrow}+Y^{-\rightarrow}-Y^{+\leftarrow}-Y^{-\leftarrow}}{Y^{+\rightarrow}+Y^{-\rightarrow}+Y^{+\leftarrow}+Y^{-\leftarrow}} \\
A_{L U}^{\mathscr{g}}(\phi) & =\frac{Y^{+\rightarrow}-Y^{-\rightarrow}-Y^{+\leftarrow}+Y^{-\leftarrow}}{Y^{+\rightarrow}+Y^{-\rightarrow}+Y^{+\leftarrow}+Y^{-\leftarrow}}
\end{aligned}
$$

with $Y^{+(-) \rightarrow(\leftarrow)}$ being the yield of DVCS events with positron (electron) beam polarized parallel (anti-parallel) to the beam direction. Using the maximum likelihood method in combination with equations $2-4$, allows for a simultaneous extraction of all the contributing moments. The resulting moments of the beam charge asymmetry are shown in figure 2 . The amplitudes have been extracted separately as a function of the momentum transfer to the nucleon $-t$, the Bjorken scaling variable $x_{B}$ and the negative square of the 4-momentum of the virtual photon $Q^{2}$, respectively. The main contribution to the BCA is the $\cos (\phi)$ term. The accuracy of the measurement allows for a rejection of certain GPD models and their parameters. In particular, the usage of a D-term in the VGG model $[10,12]$ is disfavored (the $t$-dependence in the VGG calculation shown here is modeled in a Regge-motivated ansatz), as well as a factorized ansatz for the $t$ dependence in both the VGG model and the dual model [11]. In the case of the BSA (not shown) the VGG model overshoots the data for the $\sin (\phi)$ moment of the interference term by almost a factor of two and this is the case regardless of the anstaz being taken for the $t$-dependence. A similar behavior is expected from the Dual model [14]. These examples show that the HERMES results on DVCS provide valuable information to reject GPD models and can serve as guidance in future model developments. 


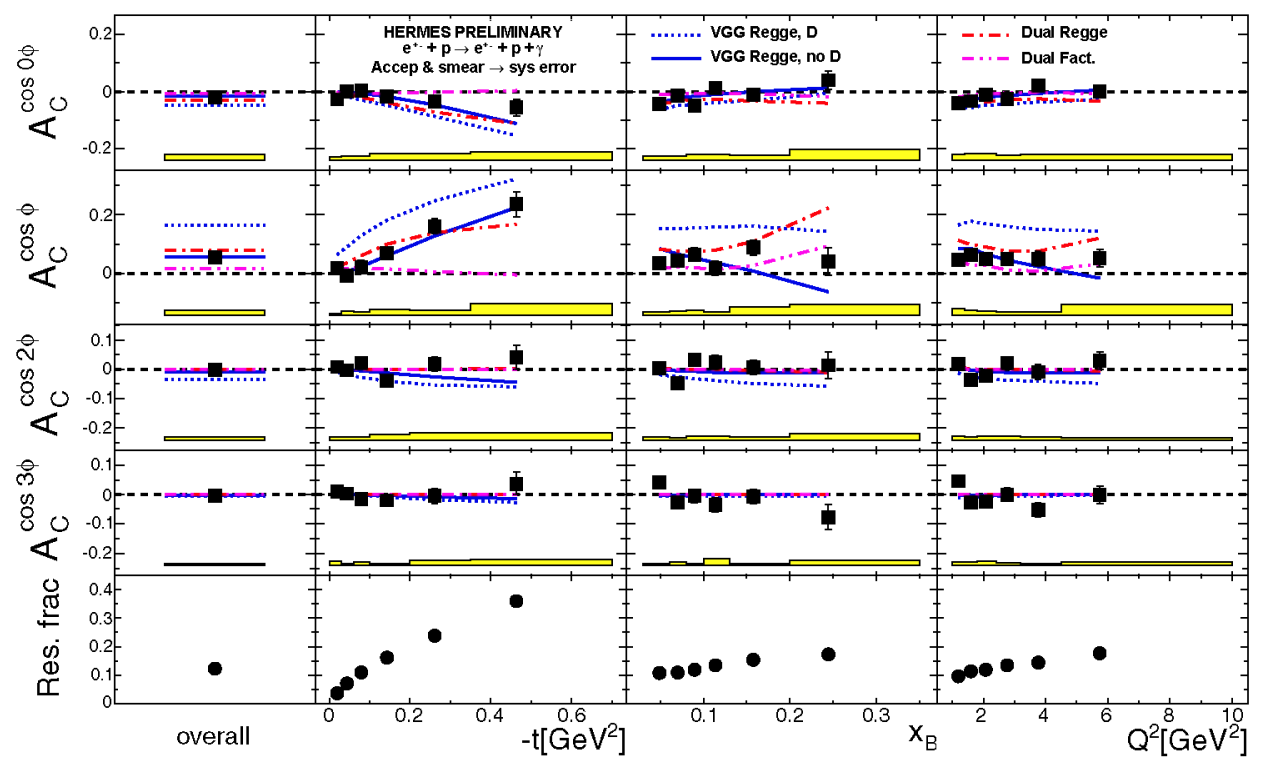

FIGURE 2. Moments of the beam charge asymmetry obtained from a maximum likelihood fit to the data.

\section{GPD E}

All observables discussed in the previous section are sensitive to the GPD $H$. In order to extract information about the total angular momentum of quarks in the nucleon using the Ji relation [2] it is necessary to measure observables that are sensitive to the GPD $E$. One of the rare observables in the HERMES regime, where the contribution from the GPD $E$ is not kinematically suppressed, is the transverse target spin asymmetry (TTSA). This observable requires a transversely polarized nucleon target. The HERMES experiment used such a target between the years 2002 and 2006 in combination with polarized electron and positron beams. Similar to the case with longitudinal beam polarization as given by equation 3 , the cross section with respect to the transverse target polarization can be written as

$$
\frac{\sigma_{U T}}{\sigma_{U U}}=1+e_{l} \cdot A_{C}\left(\phi, \phi_{S}\right)+P_{T} \cdot A_{U T}^{D V C S}\left(\phi, \phi_{S}\right)+e_{l} \cdot P_{T} \cdot A_{U T}^{I}\left(\phi, \phi_{S}\right),
$$

where the subscript $T$ refers to a transversely polarized target. As a consequence of the transverse target polarization an additional azimuthal angle $\phi_{S}$ is introduced, that defines the angle between the lepton scattering plane and the polarization vector of the target nucleon. Similarly to equation 4 three asymmetries can be calculated. Equation 2 for the Fourier terms needs to be extended to include transverse target contributions [13]. In particular, the interference term will lead to a $\sin \left(\phi-\phi_{S}\right) \cdot \cos (\phi)$ modulation of the cross 


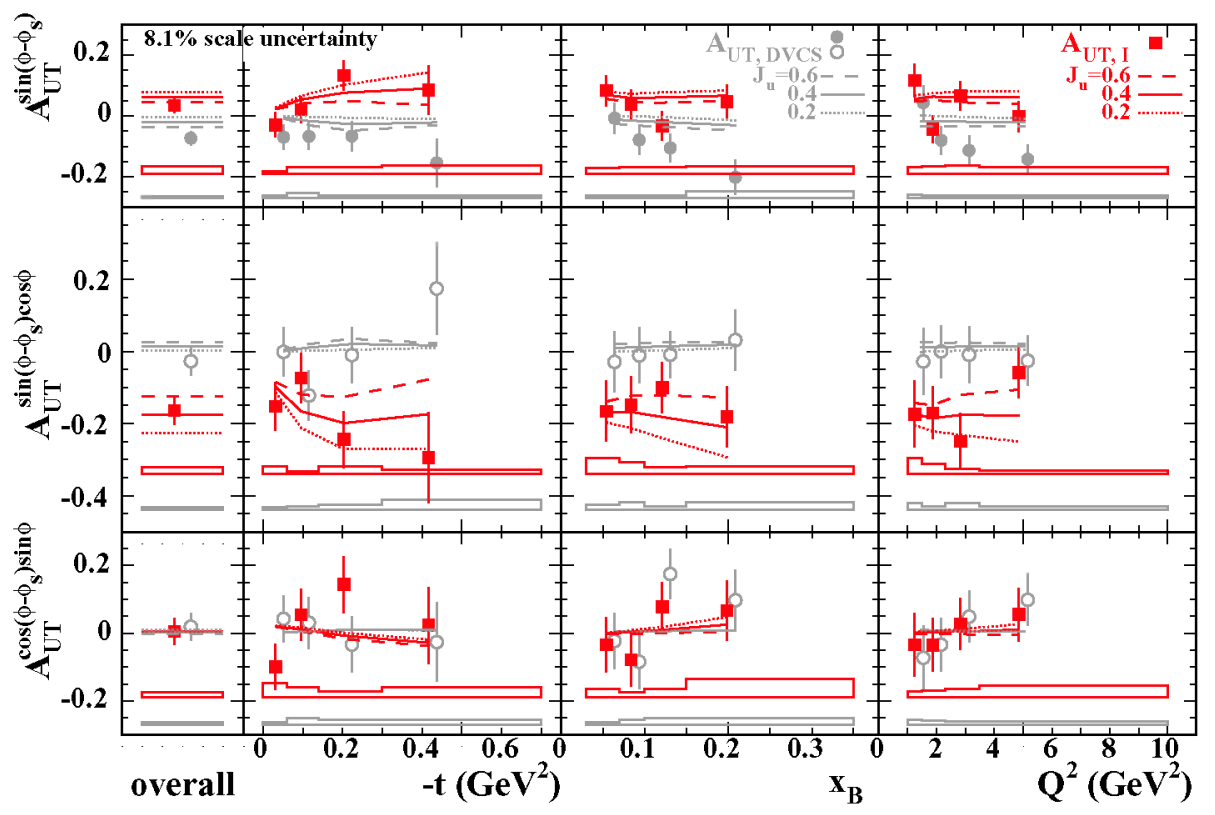

FIGURE 3. Moments of TTSA for the DVCS and interference term obtained from a maximum likelihood fit to the data.

section that can be projected out by the TTSA and is proportional to $\operatorname{Im}\left[F_{2} \mathscr{H}-F_{1} \mathscr{E}\right]$. $F_{1}$ and $F_{2}$ are the Dirac and Pauli form factors and $\mathscr{E}$ is the CFF of $E$. The full transverse data set is analyzed using the maximum likelihood method in the same way as shown above. The resulting $\phi$ and $\phi_{S}$ dependent modulations from the DVCS and interference terms are shown in figure 3 . As in the previous case, these amplitudes have been extracted as a function of $-t, x_{B}$ and $Q^{2}$. The squares in figure 3 represent the moments from the interference term, while the circles represent those from the pure DVCS term. The filled symbols are related to twist- 2 contributions while the open circles are related to twist- 3 terms. The most important moment related to the interference term, is the $\sin \left(\phi-\phi_{S}\right) \cdot \cos (\phi)$ moment and it is significantly different from zero. The three different curves, solid, dotted and dashed, represent VGG-model calculations using different values for the total angular momentum of the $u$-quark $J_{u}$, while leaving the $d$ quark contribution at zero. Such comparisons give an impression to which degree the current data set can help to understand the contribution from orbital angular momenta of quarks to the spin of nucleon.

\section{Deeply Virtual Compton Scattering on Nuclei}

The HERMES experiment collected also data with a deuterium target and other heavier nuclei: $\mathrm{He}, \mathrm{N}, \mathrm{Ne}, \mathrm{Kr}, \mathrm{Xe}$. At small values of $-t$ coherent processes dominate the 
cross section, while at larger values the incoherent scattering on protons and neutrons dominates. Comparing results on nuclei to those on hydrogen allows to investigate nuclear dependencies. Data at low $-t$ is selected in such a way that the mean value of $Q^{2}$ and $x_{B}$ of each data sample on all different targets is approximately the same. This approach however results in different combinations of coherent to incoherent contributions in the data sample for different nuclear targets. Therefore the term "coherent enriched" has been created to emphasize the fact that the data sample is a mixture of coherent and incoherent processes and its ratio depends on the nuclear target while keeping the average kinematic variables fixed. The resulting ratios for the data samples of the incoherent enriched part to that of hydrogen are consistent with unity while for the coherent enriched part the ratios are systematically larger than unity for all nuclear targets. The fit value found for the coherent enriched sample is consistent with a very basic prediction of $5 / 3$ for spin- 0 and spin- $1 / 2$ targets, based on the ratio of the involved valence-quark charges squared [15], as well as with calculations done specifically for neon and krypton [16].

\section{EXCLUSIVE MESON PRODUCTION}

Requiring a meson instead of a hard photon in the final state provides an independent process to study GPDs. In the case of exclusive pseudo-scalar meson production $\left(\pi^{+}, \pi^{o}\right)$ observables are sensitive to the polarized GPDs $\tilde{H}$ and $\tilde{E}$, while in the case of vector meson production $(\rho, \phi, \omega)$ observables are sensitive to the GPDs $H$ and $E$. These processes are somewhat more complicated than DVCS because the vertex of the outgoing meson involves a soft part as the struck quark has to fragment into a meson. In addition factorization is proven to hold only for longitudinal virtual photons, contrary to DVCS where it is also proven for transverse photons. In the case of the $\rho^{\circ}$ an L/T separation is feasible when assuming s-channel helicity conservation and separating longitudinal and transverse $\rho^{o}$-mesons by their angular decay distributions.

\section{REFERENCES}

1. D. Müller, et al., Fortschr. Phys., 42101 (1994).

2. X. Ji, Rev. Letters, 78610 (1997); Phys. Rev, D55 7114 (1997).

3. A. V. Radyushkin, Phys. Lett, B380 417 (1996); Phys. Rev., D56 5524 (1997).

4. M. Burkardt, Phys. Rev., D62 071503 (2000); Erratum2̌013ibid. D66 119903 (2002).

5. A. V. Belitsky and D. Müller, Nucl. Phys., A711 118 (2002).

6. P. Ralston and B. Pire, Phys. Rev, D66 111501 (2002).

7. K. Ackerstaff et al., Nucl. Instr: and Meth. A417 230 (1998).

8. M. Diehl et al., Phys. Lett. B411 193 (1997).

9. A. V. Belitsky, D. Müller and A. Kirchner, Nucl. Phys. B629 323 (2002).

10. M. Vanderhaeghen, P. A. M. Guichon and M. Guidal, Phys. Rev. D60 094017 (1999).

11. V. Guzey and T. Teckentrup, Phys. Rev. D74 054027 (2006).

12. K. Goeke, M. V. Polyakov and M. Vanderhaeghen, Prog. Part. Nucl. Phys. 47401 (2001).

13. M. Diehl, Phys. Rept. 38841 (2003).

14. V. Guzey and T. Teckentrup, hep-ph 0810.3899

15. A. Kirchner and D. Müller, Eur. Phys. J. C32 347 (2003).

16. V. Guzey and M. Strikman, Phys. Rev. C68 015204 (2003). 